\title{
Philosophiques
}

\section{L’art de la séparation}

\section{Jocelyne Couture}

Volume 19, numéro 2, automne 1992

Une nation peut-elle se donner la constitution de son choix?

URI : https://id.erudit.org/iderudit/027190ar

DOI : https://doi.org/10.7202/027190ar

Aller au sommaire du numéro

Éditeur(s)

Société de philosophie du Québec

ISSN

0316-2923 (imprimé)

1492-1391 (numérique)

Découvrir la revue

Citer ce document

Couture, J. (1992). L’art de la séparation. Philosophiques, 19(2), 41-54.

https://doi.org/10.7202/027190ar d'utilisation que vous pouvez consulter en ligne.

https://apropos.erudit.org/fr/usagers/politique-dutilisation/ 


\title{
L'ART DE LA SÉPARATION ${ }^{\mathrm{I}}$
}

\author{
par \\ Jocelyne Couture
}

En exergue d'un livre de Daniel Dennett on trouve cette citation de James Mc Cawley: « Le philosophe est celui qui, participant à un débat sur la peine de mort, offre une communication sur le paradoxe du pendu. ${ }^{2}$ Il est sans doute vrai que les philosophes ont quelquefois une façon bien imprévisible - c'est le moins que l'on puisse dire - de s'attaquer aux problèmes qu'on leur soumet, surtout - et ceci n'est pas une coïncidence - lorsque ces problèmes sont de nature pratique. Tout en faisant de mon mieux pour échapper à ce penchant, je n' ai quand même pas pu résister à la tentation d'amorcer ma réponse à la question qui nous réunit ici: « Une nation peut-elle se donner la Constitution de son choix? » par quelques considérations de nature sémantique.

Cette question, ceux qui en sont responsables me pardonneront bien mon impertinence, m'est apparue bizarre, elle évoque pour moi des questions comme: 'Un célibataire peut-il être non mariê?' ou encore, 'Une femme peut-elle être de sexe féminin?'. Tout comme un cèlibataire se reconnaît au fait qu'il soit non marié, une nation, à ce qu'il me semble, se reconnaît à ce qu'elle puisse, en toute légitimité, se donner la Constitution de son choix. Il serait bien futile, cependant, de prêtendre qu'un

I. Michael Walzer a utilisé l'expression dans un contexte différent. Voir: $\mathbf{M}$ Walzer, « Liberalism and the Art of Separation », Political Theory, Vol I2, $N_{3}$, Août 1984, pp. 315-52.

2. Daniel Dennett: Elbow Room, Bradford Book, Cambridge Mass., MIT Press, 1984. 
bon dictionnaire pourrait suffire à montrer que la première question, tout comme les secondes, révèle une mauvaise connaissance du français. Les usages différents ici, peuvent recouvrir des conceptions différentes de ce qu'est une nation.

Et je quitterai maintenant le terrain semantique pour arguer, non pas du caractère analytique de l'expression 'nation pourvue d'une constitution de son choix', mais pour arguer, dans une perspective carrement normative, que la seule conception de la nation qui soit acceptable est celle où les conditions d'existence d'une nation impliquent l'existence effective d'une constitution choisie par elle. Jarguerai, plus précisément, que cette conception de la nation, contrairement à celles qui feraient de la nation une entité indépendante de l'existence d'une constitution ou, si l'on veut, indépendante de l'existence d'un État qui en réponde, est la seule qui soit justifiable du point de vue philosophique et que, dans un contexte moderne, c'est-à-dire, grosso modo, postmédiéval, elle est la seule qui puisse être justifiée du point de vue des réalités politiques et sociales qu'elle recouvre. Je soutiendrai que l'histoire passée et présente du nationalisme quèbécois s'est enferrée dans une séparation conceptuelle et politique entre la nation et l'État. Je terminerai par quelques remarques concernant le sens que revêt l'expression « de son choix » dans la conception de la nation que je me propose de défendre ici.

\section{LA NATION 'vírITABLE' VS L'ÉtTAT 'EFFICACE'}

Les organisateurs de ce Colloque nous invitent à réfléchir ou à nous prononcer, ou à faire les deux à la fois, sur les circonstances dans lesquelles il est possible de se donner une constitution et ils nous invitent peut-être surtout à réfléchir ou à nous prononcer, ou à faire les deux à la fois, sur la nature des choix possibles en matière constitutionnelle.

Bien sûr la possibilité dont il est question ici n'est pas une possibilité au sens logique du terme, et elle ne renvoie pas non plus à la capacité physique, psychologique ou intellectuelle de se donner une constitution et de faire, en cette matière, des choix éclairés. La possibilité dont il est question ici s'entend dans le domaine normatif et au sens de ce qui est permis; dans quelles circonstances, dans quelle mesure, et au nom de quels critères, l'institution d'une constitution, qui consacre l'autorité et la suprématie dans un espace social donné d'un ensemble de principes d'organisation sociale, peut-elle être justifiée? La question se pose ainsi comme un problème de légitimation d'un pouvoir 
et c'est sur ce terrain que j'édifierai mon désaccord avec ce qu'elle présuppose.

Je partirai de trois exemples, illustrant trois façons d'aborder la question de la légitimité d'une organisation sociale autoritaire. Deux de ces exemples sont tirés de l'histoire de la philosophie politique et proposent des modèles dont je soutiendrai, premièrement, qu'ils sont procéduralement et substantiellement inadequats et, deuxièmement, qu'ils conduisent, en opposant chacun à sa manière l'Etat et la nation, à des impasses comparables à celles dont nous faisons maintenant l'expérience. Le troisième modèle, que je défendrai, vient d'un sociologue: Max Weber. Je commencerai par les philosophes. Mais je prendrai tout de suite la précaution de dire que je n'ai ni la prétention ni l'intention de m'engager dans une exégèse des trois auteurs dont il sera question et que c'est à titre illustratif, et pour ce qu'ils suggèrent davantage que pour ce qu'ils entendent peut-être soutenir, qu'il en sera question.

Au début du Contrat Social, Jean-Jacques Rousseau déclare

L'homme est né libre et partout il est dans les fers. I...l Comment ce changement s'est-il fait? Je l'ignore. Qu'est-ce qui peut le rendre légitime? Je crois pouvoir résoudre cette question.l... l'ordre social est un droit sacré qui sert de base à tous les autres. Cependant, ce droit ne vient pas de la nature; il est donc fonde sur des conventions. Il s'agit de savoir quelles sont ces conventions ${ }^{3}$.

Si l'ordre social est conventionnel, il n'en n'est pas pour autant arbitraire, selon Rousseau, et c'est de la légitimité des conventions qui le définissent qu'il tire sa propre justification. Les conventions seront légitimes, au sens de Rousseau, si elles permettent d'atteindre des objectifs clairement et librement conçus, répondant eux-mèmes aux volontès d'une communauté donnée. Selon Rousseau les conventions sont ou non légitimes selon qu'elles reflètent ou non cette volonté par quoi s'exprime l'essence de la communauté.

J’estime que Rousseau est un penseur de la vérité en matière de légitimité. Non pas d'une vérité absolue, transcendante et universelle bien sûr, mais de la vérité d'une communauté, des conditions objectives qui en font une communauté et des aspirations qui en constituent l'essence.

Rousseau, on le sait, ètait pessimiste quant à la légitimité des États qui lui étaient contemporains. Non pas qu'il ait

3. Jean-Jacques Rousseau, Du Contrat social, Paris, J. Roussel, Classiques Garnier (Bordas), I989, p. 250. 
constaté que la plupart d'entre eux ètaient basés sur les décisions unilatérales d'un petit nombre d'individus plutôt que sur les volontés communes, mais bien plutôt, nous dit Rousseau en substance, parce que trop peu de pays présentent les caractères d'une communauté authentique. Autrement dit, c'est moins l'absence d'un assentiment général et publiquement manifesté qui est en cause, que la capacité des sociétés à dire elles-mêmes le vrai en ce qui les concerne. Derrière les volontés communes déclarées ou exprimées, se profile toujours, pour Rousseau, le spectre des volontés communes réelles; celles que pourraient exprimer des collectivités qui n'ont subi ni ne subissent quelque forme que ce soit de coercition, d'influences extérieures susceptibles d'infléchir leurs préférences réelles et qui ont su prendre du recul face aux coutumes et traditions ancestrales génératrices d'iniquités et autres travers sociaux.

La principale difficulté de l'approche rousseauiste est évidemment d'assujettir la légitimité des conventions à la découverte d'une vérité inaccessible; on pourra toujours douter, et avec raison, de l'aptitude d'une socièté à se définir elle-même en faisant abstraction des circonstances où elle s'exprime et des conventions qui marquent déjà l'existence de cette société. Et même à supposer que l'essence d'une communauté authentique puisse ainsi être découverte, on pourra encore douter de la pertinence qu'elle peut présenter pour une société actuelle et pour les conventions que dans un contexte actuel, cette société pourrait vouloir se donner. L'objection qu'on pourrait fondamentalement adresser à Rousseau, c'est que tout en posant d'un côté l'autorité de la volonté commune en matière de conventions, il lui dénie de l'autre côté, et en la confrontant à une vérité qui lui est extérieure, toute capacité effective de faire valoir sa propre autorité en ce qui la concerne. On comprend bien que la quête de vérité en matière de légitimité puisse engendrer le pessimisme quant à la légitimité des États actuels, mais elle devrait aussi engendrer, paradoxalement, le pessimisme des collectivités. A suivre Rousseau sur cette voie, celle qui nous conduit à concevoir séparément la communauté d'abord, puis les conventions qui définissent un ordre social donné, on devrait conclure à l'impossibilitė pure et simple de légitimer des conventions, mais on devrait aussi conclure que les collectivités ou les sociétés en tant que telles ne peuvent guère contribuer à déterminer l'ordre social auquel tous ont droit.

Une deuxième façon de concevoir la légitimité des conventions est suggèrée par Hobbes. Hobbes conçoit les règles 
qui déterminent un ordre social donné comme relevant des décisions d'une instance - le Souverain ou le gouvernement qui doit elle-même son autorité en cette matière à ce qu'une assemblée d'individus lui ait confié la responsabilité - et le pouvoir corrèlatif - de réaliser un but ponctuel. Un tel but, pour Hobbes par exemple, serait de faire régner la paix sur un territoire donné en contrôlant tout ce qui peut, de l'extérieur ou de l'intérieur, y faire obstacle. Le pouvoir exercé par le Souverain est strictement délimité par les fonctions qui lui ont été confiées mais à l'intérieur de ces fonctions il a une autorité absolue. Il n'a le pouvoir d'édicter des règles que tant et aussi longtemps qu'elles sont efficaces en regard des objectifs limités qu'on lui a assignés, mais toute règle qu'il lui plaira d'édicter à cette fin est légitime du fait même qu'il l'édicte.

Hobbes est, à plusieurs égards, l'antithèse de Rousseau. Pour Hobbes, les constitutions n'ont pas pour but de favoriser l'épanouissement des communautés; elles sont plutôt dictées par la commodité et par la perspective des avantages qui découlent du fait d'avoir un ordre social quel qu'il soit; elles remplissent une fonction que l'on pourrait qualifier d'utilitaire, relièe aux désirs les plus élémentaires des individus: celui de vive en paix et d'éviter le chaos. Pour Hobbes, ce ne sont pas les communautés qui se donnent des constitutions, mais au contraire, les constitutions qui creeent des associations d'individus unis les uns aux autres par leur semblable soumission à un même ensemble de lois. Hobbes n'est pas un penseur de la vérité en matière de légitimité; il est un penseur de l'efficacitè.

Sa conception de ce qu'est une constitution légitime présente aussi des difficultés qui sont l'exact opposé de celles qu'on remarquait chez Rousseau. Alors que le critère de légitimité chez Rousseau - l'authenticité de la volonté communautaire - est trop restrictif en matière de conventions susceptibles d'être légitimées, le critère de Hobbes, - l'efficacité dans la garantie de biens élémentaires - ne l'est pas assez et risque de trouver légitimes trop de constitutions, incluant des constitutions excessivement autoritaires et possiblement conflictuelles en regard des intérêts que peuvent aussi manifester les citoyens pour des biens moins élémentaires. A suivre Hobbes sur cette voie, celle qui nous invite à concevoir séparément l'État d'abord, puis l'organisation sociale qui en résulte, on devrait conclure à l'impossibilité pure et simple, pour une nation ou un peuple uni par un projet commun de vie en société, de se donner un État efficace. On n'a pas besoin d'évoquer l'âme souffrante des 
communautés mises en demeure de marchander leurs modes de vie propres contre la survie physique de leurs membres pour concevoir qu'une constitution à la Hobbes risque d'engendrer ce qu'elle cherche précisément à éviter, à savoir le chaos social. Un souverain aux vues étroites et disposant d'un pouvoir absolu s'expose à l'instabilité. Et Hobbes bien sûr y a pense, qui ne concevait point l'exercice du pouvoir en l'absence d'un appareil cœercitif robuste.

Pour le sociologue Max Weber, et ceci sera mon troisième et dernier exemple, la question de la légitimité devrait faire place à celle de la croyance en la légitimité. Une constitution serait justifiée, autrement dit, dès lors que la collectivité qu'elle régit croit en sa légitimité.

Cette position evoquera pour plusieurs, et non sans raison peut-être, le spectre des « appareils idéologiques d'Etat » par lesquels un pouvoir - n'importe quel pouvoir - peut aniver à s'auto-justifier et grâce auxquels une société - $n^{\prime}$ importe quelle socièté - peut être conduite aux limites de l'injustifiable. On a bien sûr critiqué Weber pour son cynisme à l'endroit de l'exercice du pouvoir et à l'endroit, aussi, de l'avenir des sociétés humaines. Je pense que ces critiques, cependant, ont accordé beaucoup d'importance aux pouvoirs maléfiques de l'idéologie et peu à la nature de ce qu'est une croyance.

Une croyance, premièrement, n'existe pas isolément; elle fait partie d'un système qui, chez des individus minimalement rationnels, présente un degré raisonnable de cohérence. On peut supposer qu'une croyance en la légitimité d'une constitution donnee s'articulera d'abord sur des croyances concernant, d'une façon plus générale, la légitimité des constitutions; elle se fonderait ainsi sur une autre croyance, à savoir que la constitution jugée légitime peut être reconnue comme telle par ceux qui se sont donné des constitutions différentes. On peut de plus supposer qu'une croyance en la légitimité d'une constitution donnée s'articulera aussi sur certaines croyances portant d'abord sur les aspirations et les désirs de la collectivité concernée par cette constitution et, ensuite, sur le degré d'attachement de cette collectivité à ladite constitution; elle se baserait ainsi sur une autre croyance, à savoir que la constitution jugée légitime est reconnue comme telle par les principaux intéressés. Une croyance cohérente en la légitimité d'une constitution donnée s'appuiera donc sur des standards propres aux membres d'une collectivité en autant que ces standards sont compatibles avec des standards 
de légitimité plus généralement admis au sein d'une communauté internationale.

Une croyance rationnelle, deuxièmement, cherche à s'appuyer sur des données factuelles. Quand elle porte sur les croyances, désirs et aspirations d'autrui, elle tient pour pertinents ces croyances, désirs et aspirations qu'autrui entretient avec suffisamment de détermination pour les manifester explicitement et adopter les comportements qui y correspondent. Lorsqu'un individu croit rationnellement qu'une constitution donnée serait tenue pour légitime par ceux qui se sont donné des constitutions différentes, il s'appuie sur les normes actuellement en vigueur en vertu d'autres constitutions. Et lorsqu'il croit qu'une constitution est jugée légitime par la collectivité directement concernée par cette constitution, il base sa croyance sur des attitudes publiquement manifestées par une grande majorité des membres de cette collectivité.

Une croyance ainsi entendue suppose l'adoption de critères qui, s'ils ne peuvent pas prétendre à l'objectivitè et à l'universalité au sens strict, résument néanmoins les jugements généralement tenus pour corrects en cette matière. Une croyance ainsi entendue est relativement à l'abri des influences partiales et elle peut présenter une résistance considérable face aux manipulations idéologiques.

Il me semble qu'une constitution qui suscite une croyance rationnelle en sa légitimité, au sein d'une collectivité donnée, est une constitution justifiée. Elle l'est puisqu'elle répond aux aspirations et aux désirs de cette collectivité; à ces désirs et à ces aspirations qui se manifestent avec suffisamment de force et de détermination chez les membres de cette collectivité pour qu'ils soient prêts à accepter de se plier aux contraintes plus formelles d'un ordre social basé sur ces valeurs. Elle l'est aussi puisqu'en remportant l'adhésion d'individus qui manifestent ce degré de détermination elle garantit la stabilité de cet ordre social et par conséquent la consolidation unifiée et à long terme d'objectifs communs. Et elle l'est ensuite parce qu'en satisfaisant aux standards politiques et sociaux admis au sein d'une communauté internationale, elle confirme, et renforce, les capacités d'autonomie et d'autodétermination d'une collectivité.

Et il me semble de plus qu'une constitution dont la légitimité demeure douteuse aux yeux de la collectivité qu'elle concerne ne peut pas être justifiée. Une constitution à laquelle on ne croit pas est une constitution qui ne satisfait pas aux 
conceptions que se fait une collectivité des modes de vie désirables pour elle-même et pour les autres collectivités. C'est une constitution qui, de ce fait même, se voue ou bien au chaos et à l'instabilité ou bien à l'abus de pouvoir qui guette une constitution à la Hobbes; c'est une constitution qui discréditera, par conséquent, la collectivité qu'elle devrait représenter. Comment une constitution à laquelle on ne croit pas pourrait-elle être justifiée?

Mais à suivre Weber sur cette voie, celle qui nous invite à fonder la légitimité d'une constitution sur les croyances rationnelles d'une collectivitè, c'est-à-dire sur des croyances cohérentes, effectivement manifestées, il serait impossible de concevoir séparément l'exercice du pouvoir et la volonté commune; il serait impossible de concevoir séparément l'État et la nation.

\section{LE NATIONALISME QUÉBÉCOIS}

L'histoire du nationalisme québécois a connu, et connaît encore, ses penseurs de la vérité. Il y en a même deux sortes. Premièrement, il y a ceux qui nous ont exhorté, et nous exhortent encore, à partir à la découverte de notre identité en tant que nation, à dire ce qui nous est spécifique et ce qui nous distingue en tant que peuple authentique, étant bien entendu qu'ensuite, mais ensuite seulement, nous serions en mesure de concevoir clairement ce que nous voulons être et peut-être enfin, prendre les moyens de le devenir. Deuxièmement, il y a ceux qui nous ont invité, à grand renfort de Plaines d'Abraham et de Confédération canadienne, à trouver dans l'histoire de notre nation, l'entière justification de notre actuelle volonté politique. Ces penseurs de la vérité (des deux sortes) nous ont d'une main ferme conduit aux impasses que l'on a déjà remarquees chez Rousseau.

Partant de l'idée que nous formions une nation, les penseurs de la vérité nous ont en même temps affirmé que cela ne suffisait pas, qu'il fallait encore rechercher une vérité plus profonde et soi-disant plus convaincante: celle de notre essence ou celle de nos déboires historiques. Ils ont tenté de nous convaincre que la légitimité de nos revendications actuelles, plutôt que de trouver sa preuve dans notre présente détermination commune, dépendait strictement de ces vérités, toujours contestables et constamment rẻinterprétables. Ils nous ont placé sur la voie des vérités inaccessibles et, souvent avec la meilleure volonté du monde, j'en conviens, ils nous ont incité à nous satisfaire d'une idée, celle que nous formions une nation, et à nous désinvestir de ce 
qui a pu nous permettre d'exister en tant que nation, à savoir, la capacité de s'affirmer pour et par nous-mêmes. C'est ainsi qu'en tant que « nation », nous devions néanmoins nous demander si nous pouvons nous donner la constitution de notre choix.

Nous avons connu aussi des penseurs de l'efficacité. De ceux qui nous ont assuré que tout ce dont nous avions besoin, nation ou pas, c'était d'une agence protectrice forte, capable de faire régner la paix et la prospérité sur son territoire en contrôlant tout ce qui peut, de l'extérieur ou de l'intérieur, y faire obstacle. Que ce dont nous avions besoin c'était d'un Souverain au bras long, qu'importe ses courtes vues, exclusivement voué à des fonctions utilitaires. On nous a aussi laissé entendre que si nous voulions suivre le cours de l'histoire, c'est une collectivitè réunie par le hasard et la nécessité qui devrait nous tenir lieu de nation. Voilà qu'à côté de la nation sans État que nous proposent les penseurs de la vérité, on nous propose un Etat sans nation; le giron confédérationnel, apparemment, nous siérait bien.

Mais nous ne serions pas nécessairement dans un dilemme, car les penseurs de la vérité et ceux de l'efficacité, après avoir soigneusement séparé la nation de l'État, s'entendent parfois à merveille pour effectuer la réunion de la « nation authentique » et de « l'État protecteur ». Les premiers seraient rassurés de posséder un Etat, même si ce n'est pas exactement celui que nous aurions voulu - mais qui sait ce que nous aurions finalement voulu? - et ils seraient ravis pour peu que l'on nous reconnaisse, au sein d'une collectivité réunie par le hasard et la nécessité, une différence dont la preuve - et pour cause - leur a toujours échappé. De plus, une entité aussi vivace que la « nation » québécoise n'aurait pas vraiment besoin d'un État qui lui soit propre; elle ne court aucun risque à se rallier à un État-parapluie à la condition, bien entendu, de réfléchir de temps en temps à son identité et à son histoire. Les penseurs de l'efficacité, de leur côté, ne dédaignent pas d'ajouter au blason de leur État sans nation les couleurs d'une vie communautaire; après tout, une agence protectrice doit aussi pouvoir faire face à la compétition si elle veut survive et ce n'est pas si souvent qu'on entend l'écho d'une communauté sur ce continent. Il faut bien dire aussi que cette supériorité peut être acquise à moindre frais; l'autoritè du Souverain n'a pas grand chose à craindre d'une collectivité qui sait si bien se contenter d'une “ idée » de nation, et qu'on peut toujours, s'il en est besoin, renvoyer à ses exercices d'introspection. La nation sans État et l'État sans nation sont apparemment faits l'un pour l'autre. 
La question est maintenant de savoir ce que nous croyons. Les Québecois, à ce qu'il me semble, ont acquis un certain degré de confiance en leurs propres modes de vie. Confiance d'abord, au sens où ils en ont pris conscience; et non pas en tant que coutumes dégénérées, réactives ou subalternes, mais en tant qu'exprimant des façons de penser et des aspirations qu'ils se sont eux-mêmes données ou qu'ils ont délibérément choisi de conserver. Confiance aussi, au sens où ils ont acquis certaines certitudes concernant la valeur sociale et humaine des rapports et des interactions que ces modes de vie impliquent. Cette confiance, je crois qu'ils l'ont acquise au jour le jour, en se tournant vers le monde qui les entoure, et en $s^{\prime} y$ impliquant. Ils l'ont acquise, cette confiance, en dépit des penseurs de la vérité qui les exhortaient plutôt à se tourner vers leur essence ou vers leur passé. La « preuve » de leur identité, les Québécois l'ont cherchée bien sûr en partant de ce qu'ils sont et de ce qu'ils ont été, mais ils l'ont trouvee, et fournie, dans le monde où ils vivent. Cette confiance, ils l'ont acquise aussi, en dépit des penseurs de l'efficacité; pendant qu'ils s'affairaient, nous dessinions, nous disaient-ils, des arabesques sur le sable; au fil du temps, les arabesques ont révélé la forme d'un projet de société capable de faire valoir dans le monde contemporain les intérêts et les aspirations des Québécois. Les Québécois ont conscience d'eux-mêmes comme d'une nation et cette conscience d'eux-mêmes s'appuie non seulement sur le fait qu'ils aient en commun une histoire, une langue et une culture, ou sur le fait qu'ils partagent des modes de vie et des aspirations valables en eux-mêmes, mais aussi sur le fait que, collectivement, ils ont eu et ont encore la capacité et la volonté de décider pour et par eux-mêmes de la place qu'ils veulent occuper dans le monde contemporain. Contrairement à Rousseau, à Hobbes, et peut-être à ceux qui ont conçu le titre du présent Colloque, les Québécois ne semblent pas trouver à ce point implausible, bizarre ou même sujette à un possible désaccord, l'idée qu'une nation soit justifiee de se donner la constitution de son choix, une constitution choisie par elle en tant que nation, en regard de ses propres objectifs et spécifiquement destinee à la réalisation de ces objectifs.

Mais les Québécois entretiennent manifestement des doutes quant aux conditions de subsistance matérielle d'un Etat québécois. Telle est du moins l'hypothèse que suggère leur hésitation à se désolidariser de l'Etat qui les encadre présentement; ce dernier, en tant qu'État axé sur l'efficacité, pourrait bien avoir la compétence requise pour garantir aux Québécois les conditions 
de leur survie en tant que nation. Les Québécois seraient-ils malgré tout sur le point de succomber à la plus subtile invention de nos grands séparateurs; seraient-ils sur le point de se rallier à l'idée que leur nation sans État dans un Etat sans nation, pourrait tenir lieu de l'État-nation qu'ils ont depuis longtemps convoité? Je pense que cet espoir serait sans fondement. Non pas qu'il soit utopique de vouloir réunir les vertus d'un authentique projet de société et celles de l'efficacité, mais bien au contraire, parce qu'il est utopique de penser qu'au plan politique, ces deux facettes de l'État-nation peuvent être conçues comme des sphères indépendantes l'une de l'autre, pouvant parallèlement et harmonieusement mener leur existence propre.

La protection que nous pourrions soi-disant rechercher auprès d'un Etat requiert minimalement la stabilité de celui-ci et il est d'usage, dans les États qui souscrivent à la tradition démocratique, d'attendre la stabilité du pouvoir de la coïncidence entre les objectifs des citoyens et ceux qui sont effectivement et avec succès poursuivis par l'État. Un État démocratiquement conçu dans la perspective de l'efficacité et dans cette seule perspective sera stable s'il accorde une priorité absolue à l'efficacité. Comment entendons-nous concilier l'organisation sociale découlant de cette priorité absolue et un projet de société qui nous serait propre? L'idée même que nous ayons nos modes de vie propres suppose que nous serons potentiellement insatisfaits de ceux qui, dans une autre perspective, seraient requis de nous. Compromettrons-nous nous-mêmes la stabilité, c'est-àdire l'efficacité que nous attendons de l'État, ou renonceronsnous, au nom de cette stabilité à nos projets de société? Nous n'aurions probablement pas le choix. Car il existe bien un autre moyen de garantir la stabilité de l'État; celui-là même que prévoyait Hobbes, et qu'en des termes atténués nous appelons ici le renforcement du Pouvoir central.

Il serait inconsistant, de la part d'un Etat axé sur l'efficacité, et sur la seule efficacité, de promouvoir ou même de tolèrer des modes de vie ou des projets de société conçus dans des perspectives diffèrentes et qui risquent, en compromettant cet objectif, de compromettre le pouvoir; en toute logique, ceux-ci devront au mieux être considérés comme des priorités de second ordre, tant au plan social qu'au plan économique, et si c'est ce que requiert dans ce cas la logique du pouvoir, alors il est utopique de penser qu'il en sera autrement. Un gouvernement qui entretient délibérément cette utopie, qui entretient délibérément l'idée qu'un État conçu pour l'efficacité peut en même temps accorder 
une priorité à des projets de société conçus dans une perspective autre, est nécessairement un gouvernement qui s'apprête à réprimer, au nom même de l'efficacité qui fonde son pouvoir, tout projet susceptible de faire obstacle à sa propre stabilité; c'est un gouvernement qui, pour maintenir son pouvoir, est prêt à se dispenser de l'adhésion volontaire de certains citoyens. Et une nation qui voudrait se gagner la protection d'un Etat en s'adjoignant un État conçu dans la seule perspective de l'efficacité est ou bien une nation qui s'adonne à l'utopie ou bien une nation qui vient de renoncer délibérément à son statut de nation et qui est prête à admettre que le moteur et le fondement des sociétés se réduisent à la survie et à la prospérité matérielle.

Une nation sans Etat plus un Etat sans nation ne font pas un État-nation. L'Art de la séparation aboutit à construire des entités conflictuelles réfractaires à toute tentative de conciliation. Ses techniques peuvent varier mais en pratique, l' Art de la séparation réussit mieux, et de façon notoire, aux penseurs de l'efficacité.

\section{LE CHOIX DES QUĖBÉCOIS}

La question qui nous réunit ici porte en elle-même sa réponse et cette réponse c'est: «non ». Lorsqu'on conçoit comme sujette à un débat la question de savoir s'il est légitime pour une nation de choisir en matière constitutionnelle, c'est que notre concept de nation a déjà été soumis à l'Art de la séparation; c'est que notre concept de nation, et celui de légitimité, supposent déjà la séparation du social et du politique. Une nation conçue dans cette perspective, Rousseau et Hobbes chacun à sa manière nous l'apprenaient déjà, ne choisit pas; elle n'a ni l'autorité ni non plus la responsabilité de définir et de réaliser un projet de socièté. Une nation conçue de cette manière s'en remet à autrui pour le choix d'une constitution.

Ce n'est pas dans ce sens que les Québécois s'identifient en tant que nation. Si les Québécois sont à la recherche d'un ordre social capable de confirmer les modes de vie auxquels ils croient tout en leur permettant d'exercer leur capacité d'autodétermination à laquelle ils croient aussi, alors ils constituent une nation dans le sens, et dans la mesure, où ils conçoivent leur existence sociale comme dependante d'une affirmation politique de leur part. Mettre en doute l'opportunité de cette affirmation politique, mettre en doute la légitimité pour les Québécois de faire un choix en matière constitutionnelle, cest mettre en doute l'opportunité, pour eux, d'être ce qu'ils sont et de devenir 
ce qu'ils veulent être. Alors que leur identité sociale est étroitement définie par la vision politique à laquelle ils adhèrent, alors que leur existence sociale implique le choix d'un projet de société, il serait aussi injuste qu'incorrect de demander à leur sujet s'ils peuvent, moralement ou physiquement, choisir. De plus, il serait aussi injuste qu'incorrect de supposer à leur sujet qu'ils ont été dépourvus, jusqu'à ce jour, de la volonté et de la capacité de s'affirmer politiquement, de supposer qu'ils n'ont formé, jusqu'à ce jour, qu'une entité sociale mythique. Il est aussi injuste qu'incorrect, lorsqu'il sagit de nation au sens où les Québécois l'entendent, de séparer les sphères sociale et politique. Une nation, entendue au sens où les Québécois l'entendent et au sens où il faut l'entendre si l'on accorde la moindre importance aux aspirations des individus, au respect de soi-même et aux conditions de légitimité du pouvoir étatique, ne peut pas ne pas choisir; le plus sûr moyen de disposer, conceptuellement ou dans les faits, d'une nation ainsi entendue, est de séparer la question de son affirmation politique de celle de son existence sociale.

Les Québécois en tant que nation, en tant que collectivité dont l'existence sociale dépend d'une affirmation politique, choisiront. Ils choisiront de réaliser la vision politique qu'ils ont eux-mêmes conçue ou ils choisiront de contribuer à la réalisation d'une vision politique conçue par d'autres. Quelle qu'elle soit, la constitution qu'ils se donneront ce faisant sera légitime du fait même qu'ils l'aient collectivement et délibérément choisie sur la base de leurs croyances rationnelles.

Les Québécois peuvent choisir de se donner la constitution adaptée au projet de société dont ils ont eux-mêmes esquissé les grandes lignes. Ils se donneront, ce faisant, un Etat québécois, le seul État capable de protéger efficacement les valeurs et les modes de vie qui inspirent ce projet de société, le seul capable de permettre sa réalisation, le seul capable de donner à ce projet sa pleine expression. Par ce choix, la nation québécoise affirmera, dans un geste politique, sa volonté de demeurer une nation, c'est-à-dire sa volonté d'inscrire, de confirmer et de déterminer, dans le contexte politique contemporain, la réalité sociale qu'elle veut représenter.

Les Québécois, en tant que nation, peuvent aussi choisir de se donner une constitution adaptée à une vision politique concue par d'autres et indifférente au projet de société qu'ils ont eux-mèmes conçu. Par ce choix, les Québécois affirmeraient, 
dans un geste politique, que cette affirmation est la dernière qu'ils veulent faire en tant que nation québécoise. Mais en tant que nation ayant le pouvoir et prenant la responsabilité de déclarer elle-même sa propre disparition, les Québécois ne pourraient pas, brandissant l'étendard de leur vérité profonde ou de leur essence, prétendre demeurer malgré tout, dorénavant, une nation. Ils rie pourraient pas, déployant le paravent de la sécurité ou de la prospérité matérielle, prétendre vouloir préserver une réalité sociale québécoise tout en s'en remettant à autrui en matière de constitution. Une nation, au sens où les Québécois l'entendent, ne peut pas choisir de ne pas choisir et si elle ne choisit pas de demeurer, c'est qu'elle a choisi de disparaître.

Departement de Philosophie

Université du Québec à Montréal 